\title{
Avaliação de pacientes submetidos a tratamento cirúrgico de hiperidrose palmar quanto à qualidade de vida e ao surgimento de hiperidrose compensatória
}

\author{
Evaluation of patients submitted to surgical treatment for palmar hyperhidrosis \\ with regard to the quality of life and to the appearance of compensatory \\ hyperhidrosis
}

\author{
Priscila Oliveira Cardoso ${ }^{1}$; Kelly C. Lacerda Rodrigues²; Karla Matos Mendes²; \\ Andy Petroianu - TCBC - MG ${ }^{3}$; Marcelo Resende ${ }^{4}$; Luiz Ronaldo Alberti- ACBC-MG ${ }^{5}$
}

\section{R E S U M O}

\begin{abstract}
Objetivo: Avaliar a qualidade de vida e a presença de compensação pós-operatória de pacientes submetidos a simpatectomia torácica. Método: Foram avaliados 50 pacientes consecutivos submetidos a tratamento cirúrgico para hiperidrose palmar. Avaliou-se: o motivo principal que levou o paciente a procurar atendimento médico, a realização prévia de tratamento clínico para hiperidrose, incidência e localização corporal de hiperidrose compensatória e sua relação com a personalidade do indivíduo e o grau de satisfação do paciente com o tratamento. Resultados: Entre todos os entrevistados, 27 (54\%) relataram que o incômodo pessoal com o suor excessivo, os levou a procurar atendimento médico; 23 (46\%) tiveram prejuízo na relação social; 22 (44\%) tiveram dificuldade relativa ao ambiente escolar, enquanto 20 (40\%) relataram que o incômodo causado pela hiperidrose nas atividades relacionadas ao trabalho levou-o a procurar atendimento médico. 21 (42\%) foram considerados tímidos pelas pessoas de seu convívio. 33 (66\%) começaram a manifestar a doença na infância (até os 12 anos); 15 (30\%) na adolescência e apenas um (2\%) na fase adulta. O calor foi responsável pela crise de suor em 17 (34\%) pacientes e a ansiedade esteve presente em 19 (38\%). Irritação gerava crise de suor em 31 (62\%). Hiperidrose compensatória ocorreu em 39 (78\%) pacientes, sendo que três (6\%) tiveram compensação plantar e 28 (56\%) no dorso e oito (16\%) simultaneamente no dorso e plantar, 46 (92\%) consideram que a qualidade de vida após a operação "melhorou". Conclusão: O tratamento cirúrgico da hiperidrose melhora a qualidade de vida, porém a hiperidrose compensatória ocorre em quase todos os pacientes.
\end{abstract}

Descritores: Simpatectomia. Hiperidrose/cirurgia. Qualidade de vida. Extremidades superiores.

\section{INTRODUÇÃO}

$\Lambda_{\text {siva }}$ hiperidrose é uma condição caracterizada pela excessiva produção de suor, podendo ser generalizada ou focal. Quando generalizada, acomete todo o corpo e pode ser parte de uma afecção preexistente, como infecção, doença endócrina ou neurológica' ${ }^{1}$. Já a hiperidrose focal é idiopática e acomete uma ou mais áreas do corpo. A hiperidrose primária ou idiopática é definida como suor excessivo além das necessidades fisiológicas, que resulta em suor profuso nas regiões palmar, plantar, axilar e facial ${ }^{2}$.

A hiperidrose é associada a angústia emocional, profissional e social, já que interfere nas atividades diárias das pessoas acometidas ${ }^{2-4}$. Esses pacientes sentem-se constrangidos em cumprimentar outras pessoas por meio de aperto de mãos e têm a necessidade de trocar as roupas duas ou mais vezes por dia devido à umidade.

O tratamento pode ser cirúrgico, tópico ou sistêmico. As operações incluem a simpatectomia torácica endoscópica, que destrói o gânglio simpático por excisão, pinçamento, transsecção e ablação com cautério ou laser. 0 procedimento cirúrgico acompanha-se de reduzida morbidade e curta internação hospitalar ${ }^{5}$. Os tratamentos alternativos ao tratamento cirúrgico incluem aplicação tópica de cloreto de alumínio, anticolinérgicos tópicos e psicoterapia. Existem também tratamentos com toxina botulínica ${ }^{6-8}$, excisão das glândulas de suor, todos com resultado limitado 2,4 .

A alta incidência de hiperidrose compensatória tem sido apontada como a maior limitação no tratamento cirúrgico da hiperidrose focal. Esse fenômeno tem sido pouco estudado e não se conhece os fatores que contribuem para

Trabalho realizado na Santa Casa de Belo Horizonte - MG.

1. Acadêmica de Medicina da UFMG; 2. Médica; 3. Professor Titular do Departamento de Cirurgia da Faculdade de Medicina da UFMG; 4. Médico Cirurgião Torácico da Santa Casa de Belo Horizonte; 5. Professor Adjunto do Departamento de Cirurgia da UFMG. 
a intensidade dessa hiperidrose e para sua nova localização. Apesar disso, há melhora dos sintomas e da qualidade de vida em pacientes com hiperidrose de membros superiores $^{9,10}$. Ao avaliarem pacientes submetidos a tratamento cirúrgico da hiperidrose, Moya et al encontraram a taxa de hiperidrose compensatória em 48,4\% deles ${ }^{11}$. Já Wilson et al, verificaram uma incidência de hiperidrose compensatória em 79\% dos pacientes. Mesmo assim, 93\% deles estavam satisfeitos com os resultados da operação e consideraram que sua qualidade de vida havia melhora$\mathrm{do}^{4,12,13}$. Previamente ao tratamento os pacientes consideravam sua qualidade de vida ruim (43\%) ou muito ruim (57\%).

Diante do hiato existente na literatura com respeito à hiperidrose compensatória, o presente estudo teve por objetivo avaliar a qualidade de vida e a presença de hiperidrose compensatória em pacientes submetidos a simpatectomia torácica.

\section{MÉTODO}

Este trabalho foi realizado de acordo com as recomendações da Declaração de Helsinque e da Resolução no 196/96 do Ministério da Saúde sobre pesquisa envolvendo seres humanos e foi aprovada pelo Comitê de Ética em Pesquisa da Santa Casa de Misericórdia de Belo Horizonte ${ }^{14}$. Todos os pacientes concordaram em participar do estudo, por meio de consentimento livre e esclarecido.

Foram avaliados 50 pacientes consecutivos submetidos a tratamento cirúrgico de hiperidrose palmar, simpatectomia torácica com excisão cirúrgica de T3 a T4, por cirurgiões torácicos da Santa Casa de Misericórdia de Belo Horizonte no período de junho de 2001 a junho de 2007. Os pacientes foram identificados por seus prontuários e convidados para esta pesquisa por meio do telefone, mas não houve seleção desta população por faixa etária, sexo ou cor da pele. O Período mínimo de acompanhamento dos pacientes no pós-operatório foi de dois anos. Após assinarem o termo de consentimento, eles responderam a um questionário, que abordou situações da vida social e pessoal antes e após a operação. A veracidade dos dados foi estimulada pela garantia de sigilo quanto à identificação dos entrevistados.

Cada paciente informou todos os motivos que o levaram à procura de tratamento cirúrgico da hiperidrose e se já havia realizado algum tipo de tratamento clínico para hiperidrose. Portanto, as informações referiram-se a uma ou mais causas adversas da hiperidrose. Foi investigada a incidência de hiperidrose compensatória, a sua relação com a personalidade do indivíduo, assim como os locais do corpo em que ela foi mais freqüente. Também foi avaliado o grau de satisfação do paciente com o tratamento.

A avaliação estatística utilizou o método descritivo de média e desvio padrão da média para a idade dos pacientes. . Para avaliar a homogeneidade da amostra e as diferenças entre os parâmetros, foram utilizados os testes qui ao quadrado e exato de Fisher. Calculou-se também o odds ratio e o intervalo de confiança das variáveis analisadas. Análise de regressão logística multivariada foi realizada para verificar a relação entre a ocorrência de hiperidrose compensatória e seus fatores preditivos. As diferenças foram consideradas significativas para valores correspondentes a $\mathrm{p}$ $<0,05$.

\section{RESULTADOS}

Foram avaliados 50 pacientes, sendo 38 (76\%) do sexo feminino e 12 (24\%) do sexo masculino. A idade dos pacientes variou entre 13 e 46 (média de 20,5 $\pm 7,5$ ) anos. A idade das mulheres variou entre 13 e 43 (21,0 \pm$6,7)$ anos e a dos homens, variou entre 14 e 46 (20,0 \pm$10,2)$ anos.

Entre todos os entrevistados, 27 (54\%) relataram que o incômodo pessoal com o suor excessivo os levou a procurar atendimento médico; 23 (46\%) pacientes tiveram prejuízo na relação social; 22 (44\%) pacientes tiveram dificuldade relativa ao ambiente escolar; 20 (40\%) relataram que o incômodo causado pela hiperidrose nas atividades relacionadas ao trabalho os levou a procurar atendimento médico, enquanto cinco (10\%) pessoas referiram problemas com discriminação (Tabela 1).

Segundo os entrevistados, 21 (42\%) deles são considerados tímidos pelas pessoas de seu convívio.

Em relação à época em que os pacientes perceberam o início do sintoma, 33 (66\%) dos pacientes começaram a manifestar a doença na infância (até os 12 anos); 15 (30\%) na adolescência (12 anos aos 18 anos) e apenas um (2\%) na fase adulta (após os 18 anos), sendo que um paciente não soube informar quando iniciou a doença. O tempo de manifestação da doença até a realização do procedimento operatório variou de um a 40 anos (média de 14,62 \pm 8,84 anos e mediana de 14,50 anos)

Dos 50 pacientes analisados, 14 (28\%) haviam realizado algum tipo de tratamento clínico específico para hiperidrose, como fórmulas tópicas de cloreto de alumínio, formaldeído e glutaraldeído, além de apare-

Tabela 1 - Motivos que levaram pacientes portadores de hiperidrose à procura de tratamento cirúrgico.

\begin{tabular}{|c|c|c|}
\hline Motivos & Número de Pacientes & $\%$ \\
\hline Incômodo pessoal & 27 & 54 \\
\hline Prejuízo social & 23 & 46 \\
\hline Dificuldade escolar & 22 & 44 \\
\hline Incômodo no trabalho & 20 & 40 \\
\hline Discriminação pela sociedade & 5 & 10 \\
\hline
\end{tabular}

Alguns pacientes revelaram mais de uma causa de desconforto decorrente da hiperidrose. 
Tabela 2 - Análise de regressão logística multivariada de variáveis relacionadas a hiperidrose compensatória.

\begin{tabular}{|c|c|c|c|c|}
\hline \multirow[t]{2}{*}{ Variável } & \multicolumn{2}{|c|}{$\begin{array}{l}\text { Hiperidrose Compensatória } \\
\text { número de pacientes }\end{array}$} & \multirow[b]{2}{*}{ Valor $\mathrm{p}$} & \multirow[b]{2}{*}{ OR } \\
\hline & Sim & Não & & \\
\hline \multicolumn{5}{|l|}{ Sexo } \\
\hline Feminino & 25 & 13 & 0,955 & 0,96 \\
\hline Masculino & 8 & 4 & & \\
\hline \multicolumn{5}{|l|}{ Incomodo trabalho } \\
\hline $\operatorname{Sim}$ & 15 & 5 & 0,678 & 0,75 \\
\hline Não & 24 & 6 & & \\
\hline \multicolumn{5}{|l|}{ Incomodo social } \\
\hline Sim & 17 & 6 & 0,523 & 0,64 \\
\hline Não & 22 & 5 & & \\
\hline \multicolumn{5}{|l|}{ Discriminação } \\
\hline Sim & 4 & 1 & 0,799 & 0,74 \\
\hline Não & 38 & 7 & & \\
\hline \multicolumn{5}{|l|}{ Desconforto escolar * } \\
\hline $\operatorname{sim}$ & 21 & 1 & 0,0089 & 11,67 \\
\hline Não & 18 & 10 & & \\
\hline \multicolumn{5}{|l|}{ Desconforto pessoal } \\
\hline Sim & 7 & 20 & 0,2611 & 2,33 \\
\hline Não & 3 & 20 & & \\
\hline \multicolumn{5}{|l|}{ Tempo até a cirurgia } \\
\hline Antes 14,5 anos & 20 & 4 & & \\
\hline Após 14,5 anos & 21 & 5 & 0,81 & 1,19 \\
\hline
\end{tabular}

OR: odds ratio

$* p=0,0089$

Ihos de iontoforese. Desses, três $(21,4 \%)$ obtiveram meIhora dos sintomas com o tratamento tópico de cloreto de alumínio.

Em relação à psicoterapia, oito (16\%) pacientes tiveram acompanhamento psicológico especializado em algum momento da vida, porém nenhum deles obteve melhora da hiperidrose primária.

Tecidos sintéticos, medo, vergonha e tristeza ou alegria desencadeavam ou pioravam a hiperidrose de três dos pacientes. O calor foi responsável pela crise de suor em 17 pacientes (34\%) e a ansiedade esteve presente em 19 pacientes (38\%). Irritação gerava crise de suor em 31 (62\%), enquanto cinco (10\%) pacientes não relacionaram a hiperidrose a fator algum físico ou emocional.

Dos 50 pacientes submetidos a simpactectomia torácica, 42 (84\%) tinham hiperidrose associada em outro local sendo 40 (80\%) à hiperidrose plantar.

Complicações operatórias foram observadas em cinco pacientes (10\%): dispnéia, anidrose, aderências pulmonares, depressão. Dessas, excetuando-se a anidrose, todas foram transitórias e não requereram reoperação.

Dos 50 pacientes submetidos à operação, 10 (20\%) tiveram persistência da hiperidrose na região palmar ou axilar; mesmo assim, sete (70\%) desses acharam que sua qualidade de vida melhorou após a operação, um (10\%) considerou sua vida inalterada e dois (20\%) relataram pio- ra da qualidade de vida e se arrependeram da operação. Das 10 pessoas em que os sintomas persistiram, quatro (40\%) continuaram com a hiperidrose palmar e cinco (50\%) passaram a ter hiperidrose axilar.

Hiperidrose compensatória ocorreu em 39 (78\%) pacientes, sendo que três (6\%) pacientes tiveram compensação plantar, 28 (56\%) no dorso e em oito (16\%) casos houve a hiperidrose compensatória em região plantar e no dorso simultaneamente. Dentre os 39 pacientes, dois $(5,1 \%)$ arrependeram-se da operação. Foi observado hiperidrose compensatória em 25 mulheres $(65,7 \%$ das mulheres) e em oito homens (66,6\% dos homens), não havendo relação entre a ocorrência de hiperidrose compensatória e o sexo $(p=0,955 ; O R=0,96,0,2<O R<$ 4,54).

A qualidade de vida antes da operação foi "boa" para 29 (58\%) pacientes, para cinco (10\%) "muito boa", para $12(24 \%)$ "ruim" e para quatro (8\%) "muito ruim". Ainda nesse aspecto, 46 (92\%) consideram que a qualidade de vida após a operação "melhorou"; para dois (4\%) continuou "inalterado" e para dois pacientes "piorou".

Dos 50 pacientes submetidos a simpatectomia, dois deles (4\%) relataram ter se arrependido do procedimento. Nesses pacientes, foram verificados persistência da hiperidrose palmar, bem como de hiperidrose compensatória em dorso, abdome e região torácica anterior em um paciente e planta do pé e abdome no outro. Em ambos os 
pacientes, houve piora da qualidade de vida em todos os aspectos estudados.

Para 23 (46,9\%) pacientes exercer atividade manual era "muito ruim" antes da operação, "ruim" para $16(32,7 \%)$ e "boa" para 10 (20,4\%) dos pacientes. Após a operação, essas atividades melhoraram para 45 (90\%) pacientes, foi "inalterado" para três (6\%) e "piorou" para dois (4\%).

Quando questionados sobre as atividades sociais (como aperto de mão e dançar) antes da operação, 21 (42\%) pacientes relataram que a prática dessas atividades era "muito ruim", 22 (44\%) "ruim", seis (12\%) "boa" e um (2\%) "muito boa". Houve melhora após o procedimento cirúrgico para 46 (92\%) pacientes, para dois (4\%) foi "inalterada" e para dois (4\%), ela "piorou".

Ao comparar a idade das pessoas que tiveram hiperidrose compensatória com as que não tiveram quando da operação, notou-se que os primeiros tinham a média de idade de $22 \pm 1,145$ anos e os pacientes sem hiperidrose compensatória tinham 24,5 $\pm 2,941$ anos $(p=0,326)$ $(\mathrm{OR}=1,31 ; 0,25<\mathrm{OR}<6,99)$.

Dos pacientes considerados tímidos pelos seus familiares ( $n=21), 17$ (81\%) tiveram hiperidrose compensatória e dos não considerados tímidos ( $n=29), 22(75,8 \%)$ também tiveram essa complicação. Não foi verificada diferença significativa entre a presença de timidez e a ocorrência de hiperidrose compensatória $(p=0,16)(O R=0,42$; $0,11<\mathrm{OR}<1,67)$.

Ao confrontar a idade dos pacientes submetidos à simpatectomia com o surgimento de hiperidrose compensatória, verificou-se que das pessoas com idade menor à da mediana de 14,5 anos $(n=25), 21(84 \%)$ tiveram hiperidrose compensatória e das com idade superior a mediana ( $n=25), 20(80 \%)$ tiveram essa adversidade, sem diferença significativa entre ambos os grupos $(p=0,71)$ OR $=1,31 ; 0,25<\mathrm{OR}<6,99$ ).

A tabela 2 mostra a análise de regressão logística multivariada de fatores relacionados ao aparecimento de hiperidrose compensatória. Observou-se que a presença de desconforto escolar motivando a indicação do procedimento cirúrgico (22 pacientes) estava relacionado a uma maior ocorrência de hiperidrose compensatória (21 pacientes) $(95,45 \%)$ quando comparado aos pacientes sem essa queixa (18 pacientes ou $64,28 \%$ ) ( $p=0,0089$ ). Não foi verificada relação entre sexo, incomodo no trabalho, incomodo social, discriminação, desconforto pessoal e tempo até o procedimento operatório com a ocorrência de hiperidrose compensatória.

\section{DISCUSSÃO}

A simpatectomia torácica tem como indicação mais freqüente a hiperidrose primária. É uma doença crônica, de etiologia complexa e atinge cerca de $1 \%$ da população, ocorrendo tanto em homens quanto em mulheres. Em nosso estudo, a maior parte dos pacientes com indica- ção operatória para tratamento de hiperidrose primária era do sexo feminino.

De acordo com Campos et al, pessoas com sudorese excessiva sofrem freqüentemente com a tensão psicossocial e restringem suas vidas privada e profissional, apresentando inclusive dificuldades escolares. Neste estudo, verificou-se que os aspectos prejudiciais dessa afecção que levaram os pacientes a procurar assistência médico-cirúrgica foram relacionados ao desconforto no trabalho, o incômodo pessoal e o desajuste no ambiente escolar ${ }^{2}$

A hiperidrose primária é considerada manifestação psicossomática, sendo também chamada de "hiperidrose emocional". Observou-se, em nosso estudo, que a maior parte dos pacientes já manifestava a doença desde a infância ou adolescência. No entanto, menos da metade dos pacientes consideravam-se tímidos ou assim eram considerados pelas pessoas que conviviam com eles. Portanto, não se pode atribuir à timidez um papel de destaque na hiperidrose, nem considerar que a hiperidrose leve a timidez. Observouse que os desencadeantes das crises de suor são quase sempre emocionais, sendo a irritação o fator gerador mais citado, embora calor esteja quase sempre envolvido ${ }^{3,5}$.

Por meio de questionários de avaliação, muitos autores relataram uma incidência alta de sudorese compensatória. Segundo Loscertales et al , hiperidrose compensatória ocorre em $67 \%$ dos casos, sendo os locais de compensação mais freqüentes o dorso e abdome (17\%), seguidos pelas coxas $(13 \%)^{4}$. No presente estudo, os locais de hiperidrose compensatória não diferiram dos encontrados por Loscertales et al, entretanto, a sua incidência diferiu em ambos os trabalhos. Segundo a literatura, tal efeito colateral é moderado e bem tolerado pelos pacientes e tanto a qualidade de vida quanto os sintomas melhoram após o tratamento operatório. Segundo Young et al há uma melhora na qualidade de vida em todos os pacientes operados. Sem o mesmo resultado excelente, os pacientes deste estudo também estiveram muito satisfeitos com a operação ${ }^{5}$. Observou-se, então, que a hiperidrose compensatória é uma conseqüência preferível à hiperidrose primária.

Um problema encontrado nesse tipo de tratamento é a impossibilidade de reversão da cirurgia nos pacientes que se arrependeram do procedimento operatório. Ainda que o grau de satisfação com o tratamento chegue a $92 \%$ dos pacientes, naqueles que permaneceram insatisfeitos não é possível restituir a condução nervosa do gânglio simpático após excisão, na tentativa de amenizar a sudorese compensatória intensa. Não obstante exista a possibilidade de reversão após clipagem do gânglio simpático, não há estudos que confirmem tal hipótese. Portanto, uma avaliação criteriosa se faz necessária nas indicações de simpatectomia torácica para tratamento de hiperidrose de membros superiores.

O tratamento cirúrgico da hiperidrose melhora a qualidade de vida, porém a hiperidrose compensatória, que ocorre em quase todos os pacientes, é muito desconfortável. 


\title{
A B S T R A C T
}

\begin{abstract}
Objectives: The purpose of this study was to assess the quality of life and the presence of compensatory hyperhidrosis of patients submitted to sympathectomy as a treatment of hyperhidrosis. Methods: Fifty consecutive patients submitted to surgical treatment for hand hyperhidrosis at the Santa Casa Hospital of Belo Horizonte were included in this investigation, according to their age, gender and skin color. These patients were studied according to the indication of surgical treatment, previous clinical therapeutics, their complaints related to hyperhydrosis, incidence of compensatory hyperhidrosis and its relation with personality and local of hyperhidrosis. The patients' satisfaction with the treatment was observed as well. Differences were considered as significant when their values were greater than the corresponding to $p<0.05$. Results: Personal discomfort with excessive perspiration was the main complaint of 27 (54\%) patients; social embarrassment occurred in 23 (46\%) of these patients; adversities at school was reported by 22 (44\%) patients; discomfort at work occurred in 20 (40\%) patients. 33 (66\%) of these patients started to feel the effects of the ailment during the childhood; 15 (30\%) when they were teen-agers (from 12 to 18 years of age) and only 1 (2\%) started this problem in the adult life (after 18 years of age). Heat was responsible for bringing on a perspiration crisis in $17(34 \%)$ of the patients and anxiety occurred in 19 (38\%). Anger or exasperation provoked crisis in 31 (62\%) patients. Compensatory hyperhidrosis occurred in 39 (78\%) of patients including 3 (6\%) compensatory perspiration on feet, 28 (56\%) on dorsum, and 8 (16\%) presented either on dorsum and feet. Despite these findings, 46 (92\%) patients considered their quality of life improved. Conclusion: Surgical treatment for hyperhidrosis improved the quality of life of our patients, and compensatory hyperhidrosis was present in most of them.
\end{abstract}

Key words: Sympathectomy. Hyperhidrosis/surgery. Quality of life. Upper extremity.

\section{REFERENCIAS}

1. Haider A, Solish N. Focal hyperhidrosis: diagnosis and management. CMJA. Can Med Assoc J. 2005; 172(1): 69-75.

2. Campos JR, Kauffman P, Werebe Ede C, Andrade Filho LO, Kusniek S, Wolosker N, Jatene FB. Quality of life, before and after thoracic sympathectomy: report on 378 operated patients. Ann Thorac Surg. 2003; 76(3): 886-91.

3. Glaser DA, Hebert AA, Pariser DM, Solish N. Primary focal hyperhidrosis: scope of the problem. Cutis. 2007; 79(5 Suppl): 5-17.

4. Loscertales J, Arroyo Tristán A, Congregado Loscertales M, Jiménez Merchán R, Girón Arjona JC, Arenas Linares C, Ayarra Jarné J. [Thoracoscopic sympathectomy for palmar hyperhidrosis: immediate results and postoperative quality of life]. Arch Bronconeumol 2004; 40(2): 67-71.

5. Young O, Neary P, Keaveny TV, Mehigan D, Sheehan S. Evaluation of the impact of transthoracic endoscopic sympathectomy on patients with palmar hyperhydrosis. Eur J Vasc Endovasc Surg. 2003; 26(6): 673-6.

6. Naumann MK, Hamm H, Lowe NJ. Botox Hyperhidrosis Clinical Study Group. Effect of botulinum toxin type A on quality of life measures in patients with excessive axillary sweating: a randomized controlled trial. Br J Dermatol. 2002; 147(6): 1218-26.

7. Tan SR, Solish N. Long-term efficacy and quality of life in the treatment of focal hyperhidrosis with botulinum toxin A. Dermatol Surg. 2002; 28(6): 495-9.

8. Glaser DA, Hebert AA, Pariser DM, Solish N. Facial hyperhidrosis: best practice recommendations and special considerations. Cutis. 2007; 79(5 Suppl): 29-32

9. Sayeed RA, Nyamekye I, Ghauri AS, Poskitt KR. Quality of life after transthoracic endoscopic sympathectomy for upper limb hyperhidrosis. Eur J Surg. 1998; 164(Suppl 580): 39-42.

10. Lau WT, Lee JD, Dang CR, Lee L. Improvement in quality of life after bilateral transthoracic endoscopic sympathectomy for palmar hyperhydrosis. Hawaii Med J. 2001; 60(5): 126-37.
11. Moya J, Ramos R, Morera R, Villalonga R, Perna V, Macia I, Ferrer $G$. [Results of high bilateral endoscopic thoracic sympathectomy and sympatholysis in the treatment of primary hyperhidrosis a study of 1016 procedures]. Arch Bronconeumol. 2006; 42(5): 2304.

12. Wilson MJ, Magee TR, Galland RB, Dehn TCB. Results of thoracoscopic sympathectomy for the treatment of axillary and palmar hyperhidrosis with respect to compensatory hyperhidrosis and dry hands. Surg Endosc. 2005; 19(2): 254-6. Epub 2004 Nov 18.

13. Kumagai K, Kawase H, Kawanishi M. Health-related quality of life after thoracoscopic sympathectomy for palmar hyperhidrosis. Ann Thorac Surg. 2005; 80(2): 461-6.

14. Petroianu A. Pesquisa em medicina. In: Petroianu A, editor. Ética, moral e deontologia médicas. Rio de Janeiro: Guanabara Koogan; 2000. p. 174-8.

Recebido em 07/07/2008

Aceito para publicação em 06/10/2008

Conflito de interesses: nenhum

Fonte de financiamento: nenhuma

\section{Como citar este artigo:}

Cardoso PC, Rodrigues KC, Mendes KM, Petroianu A, Resende M, Alberti LR. Avaliação de pacientes submetidos a tratamento cirúrgico de hiperidrose de membros superiores quanto à qualidade de vida e ao surgimento de hiperidrose compensatória. Rev Col Bras Cir. [periódico na Internet] 2009; 36(1). Disponível em URL:http://www.scielo.br/ $\mathrm{rcbc}$

Endereço para correspondência:

Prof. Andy Petroianu

E-mail: petroian@medicina.ufmg.br 